Mots. Les langages du politique

104 | 2014

Les livres de journalistes politiques

\title{
Comment Millénium fictionnalise le journalisme du
} $\mathrm{XxI}^{\mathrm{e}}$ siècle

Millénium fictionalizes twenty-first century journalism

\section{Roselyne Ringoot}

\section{(2) OpenEdition}

Journals

Édition électronique

URL : https://journals.openedition.org/mots/21582

DOI : $10.4000 /$ mots. 21582

ISSN : 1960-6001

\section{Éditeur}

ENS Éditions

\section{Édition imprimée}

Date de publication : 19 mai 2014

Pagination : 41-55

ISBN : 978-2-84788-542-2

ISSN : 0243-6450

Référence électronique

Roselyne Ringoot, "Comment Millénium fictionnalise le journalisme du xxle siècle », Mots. Les langages du politique [En ligne], 104 | 2014, mis en ligne le 19 mai 2016, consulté le 23 avril 2022. URL : http:// journals.openedition.org/mots/21582; DOl : https://doi.org/10.4000/mots.21582 


\section{Comment Millénium fictionnalise le journalisme du XXI ${ }^{e}$ siècle}

Avant que l'industrie cinématographique ne s'en saisisse, Millénium s'est imposé dans celle du livre : en 2008 , le roman suédois est traduit dans une vingtaine de langues et huit millions d'exemplaires sont vendus dans le monde, dont deux millions en France. Actes Sud lance, avec Millénium, la collection "Actes Noirs» dédiée au «polar», qui vient diversifier le catalogue de la maison d'édition réputée experte en littérature étrangère. Selon le classement Ipsos - Livres Hebdo, le premier volume de Millénium est le livre le plus vendu en 2008 et la trilogie fait partie des dix meilleures ventes ${ }^{1}$. Quelques mois après sa parution, Millénium est déclaré «roman culte» par la presse généraliste, culturelle, traditionnelle ou en ligne ${ }^{2}$. Bibliobs.com crée un dossier intitulé «Milléniumania », relatant des «paroles de lecteurs devenus accros » 3 et suscitant un nombre considérable de commentaires liés à différents articles. En 2009, le pseudo-scoop publié en guise de poisson d'avril par le site Rue89 en dit long sur la notoriété du roman: «Exclusif: nous avons retrouvé le tome IV de Millénium. Des hackers suédois ont récupéré le manuscrit dans l'ordinateur de Stieg Larsson et vont le diffuser gratuitement sur le web »4... Et le site de dévoiler à ses «riverains» quelques (faux) extraits de l'intrigue qui se situe à Paris.

Cet engouement suscité par le roman rappelle la question posée par Umberto Eco au sujet de la «machine narrative à l'origine du succès », «qui de façon singulière, est dû tant au consentement des masses qu'à l'appréciation des lecteurs les plus raffinés» (Eco, 1981, p. 84). Comme d'autres sagas publiées ces dernières décennies dans le contexte d'une industrie culturelle internationalisée, le récit de Millénium articule, au niveau élémentaire de la

1. 512800 volumes pour Millénium 1, 377300 pour Millénium 2 et 347300 pour Millénium 3.

2. Pour exemple: «Après avoir remis à son éditeur le manuscrit des trois premiers volumes de Millénium, Stieg Larsson est mort brutalement en novembre 2004, à 50 ans. Cette mort prématurée a sans doute contribué à faire de Millénium une œuvre culte mais les qualités du projet y auraient bien suffi... » Gérard Meudal, Le Monde, 12 octobre 2007.

3. http://bibliobs.nouvelobs.com/romans/20080214.BIB0787/pourquoi-aimez-vous-millenium. html (consulté le 8 novembre 2013).

4. http://www.rue89.com/2009/04/01/exclusif-nous-avons-retrouve-le-tome-4-de-millenium (consulté le 8 novembre 2013).

Sciences Po Grenoble, École de journalisme de Grenoble roselyne.ringoot@u-grenoblez.fr 
signification, l'opposition entre le bien et le mal. Mais la «machine narrative » nous intéresse ici surtout en ce qu'elle mobilise des personnages et des actions qui contribuent à fictionnaliser le journalisme contemporain. Dans ce cadre, le système axiologique qui sous-tend le récit se déploie autour de l'opposition vérité/mensonge, ou encore, révélation/secret. Millénium acquiert le statut d'« œuvre» en même temps que Stieg Larsson, journaliste peu connu en France, acquiert le statut d'écrivain, une consécration qui interroge la fabrication sociale du journalisme dans le cadre d'un succès littéraire. En tant que livres portant sur le journalisme et en tant que livres publiés par un journaliste, la série Millénium s'inscrit doublement dans la problématique de la construction symbolique de la profession.

L'éditeur français de Millénium prend soin de rappeler, en quatrième de couverture, la fonction et le média d'exercice de l'auteur, comme dans la plupart des notices biographiques consacrées aux livres de journalistes, quand bien même il s'agit ici d'un roman. L'auteury est présenté en tant que journaliste politique engagé contre l'extrême droite, cofondateur de la revue Expo, «observatoire des manifestations ordinaires du fascisme » dont il a été rédacteur en chef. On précise également qu'il a produit «des essais sur l'économie et des reportages de guerre sur l'Afrique». Ces indications font alors écho à celles qui concernent le héros journaliste, «ancien rédacteur de Millénium5, revue d'investigations sociales et économiques ». Le paratexte du premier tome suggère ainsi la gémellité de l'auteur et de sa créature, ou pour le moins, légitime l'un par l'autre en assignant une dimension journalistique à la fiction.

S'appuyant principalement sur l'identité professionnelle, la construction de l'image d'auteur tout comme la construction du personnage inscrivent le roman dans l'univers journalistique, ou plus largement dans ce que Guillaume Pinson nomme « une culture médiatique »: «Il n'est pas de culture médiatique sans mise en scène de la culture médiatique, pas de journal sans représentation du journal, et surtout pas de journalistes sans métadiscours des journalistes.» (Pinson, 2012) Dans cette perspective, nous appréhendons le récit romanesque comme un discours tenu sur la profession, en interrogeant l'héroïsation des journalistes telle qu'opérée par Stieg Larsson. Le héros, Mikael Blomkvist, incarne la catégorie du « reporter enquêteur» dont l'émergence littéraire remonte à la fin du xixe siècle (Pinson, 2010). Pour autant, le paratexte du roman induit un autre horizon d'attente que celui de ses aventures, car en tant que titre secondaire des trois volumes, Millénium érige la revue en personnage principal. Identifiant à la fois le roman et l'acteur narratif, le nom du magazine s'impose au détriment du nom du journaliste qui caractérise fré-

5. Nous utilisons l'italique pour signifier le nom du roman (Millénium) et les caractères romains pour la revue-personnage (Millénium). L'italique est toutefois conservé dans les citations où la revue est mentionnée. 
quemment les ouvrages de fiction journalistique fondés sur un héros récurrent du type Rouletabille ou Boro6.

Si l'histoire de la revue Millénium et les péripéties de Mikael Blomkvist sont intimement liées dans la narration, et si l'identité éditoriale de la revue et l'identité professionnelle du journaliste y sont interdépendantes, deux acteurs distincts sont néanmoins mis en scène. Postulant que le journalisme développe une auctorialité complexe tiraillée entre identité collective et identité individuelle, l'étude de Millénium que nous proposons a pour objectif d'analyser comment la fiction participe à la représentation de ces deux formes auctoriales. Les deux figures personnalisent l'auteur collectif incarné par Millénium et l'auteur individualisé incarné par Mikael Blomkvist. Cependant, le journalisme tel qu'imaginé dans le roman s'articule également sur un autre personnage central par qui s'opère la triangulation actorielle journal / journaliste / hackeur. Dans une approche narrative visant la procédure de fictionnalisation du journalisme, ce personnage incarné par une jeune femme nommée Lisbeth Salander s'avère d'autant plus important qu'il endosse le rôle actanciel d'adjuvant dans les enquêtes journalistiques racontées. Par ce biais, la fiction intègre la pratique du hacking dans le monde journalistique qu'elle construit et renouvelle le tandem journaliste/détective institutionnalisé dans le genre polar.

S'orientant vers la fiction au tournant d'une trajectoire dont les caractéristiques correspondent à celle d'un journaliste engagé plus qu'à celle d'un rubricard classique de l'information politique, Stieg Larsson a plusieurs publications de livres à son actif. Maoïste dans sa jeunesse, puis trotskyste et collaborateur à L'International, féministe, antiraciste et luttant contre l'extrême droite, il débute sa carrière dans le cadre du militantisme puis intègre, en 1979, la grande agence de presse Tidningarnas Telegrambyra où il exercera jusqu'en 1999, d'abord en tant que secrétaire de rédaction, puis en tant qu'illustrateur au service Images et reportages. Durant cette période, il collabore à Searchlight, mensuel antifasciste basé à Londres, s'investit dans la revue Expo créée en 1995 et commence à copublier des ouvrages7. Pour autant, cette activité éditoriale qui le confirme en tant que spécialiste de la montée de l'extrême droite en Suède a peu d'effet sur sa position au sein de Tidningarnas Telegrambyra, qu'il quitte à l'occasion d'un licenciement économique. Au début des années deux mille, Stieg Larsson publie un «manuel pour les journalistes menacés de mort», issu de sa propre expérience en tant que cible des extrémistes, et cosigne d'autres livres sur ses thèmes de prédilection ${ }^{8}$, en

6. Les aventures extraordinaires de Joseph Rouletabille, de Gaston Leroux, ou Les aventures de Boro, reporter photographe, de Dan Franck et Jean Vautrin.

7. Il publie avec Anna-Lena Lodenius Extremhögern (L'extrême droite), Oslo, Tidens Forlag, 1991, et collabore à The Skinheads International (New York, ADI, 1995) et aux Extrémismes en Europe, J.-Y Camus éd. (La Tour d’Aigues, L’Aube, 1998).

8. Deux ouvrages sous l'égide d'Expo: Sverigedemokraterna. Den nationella rörelsen [Les démocrates suédois. Le Mouvement national], avec M. Ekman, Stockholm, Ordfront, 2001, et Sverige- 
même temps qu'il entame la rédaction de Millénium. Plusieurs éléments biographiques convergent vers l'idée que l'investissement dans l'écriture d'un roman aux abords de la cinquantaine se fonde sur un sentiment de déception professionnelle9. Quoi qu'il en soit, Millénium «auctorise» Stieg Larsson sans l'extraire du cadre professionnel journalistique, et le roman met en abyme l'auctorialité journalistique en conférant à la revue et aux livres des rôles narratifs prépondérants.

\section{Millénium, un héros à part entière}

Comme indiqué dans l'introduction, le dispositif paratextuel associe le nom «Millénium » au titre principal de chaque livre (Les hommes qui n'aimaientpas les femmes, La fille qui rêvait d'un bidon d'essence et d'une allumette, La reine dans le palais des courants d'air) en procédant à la numérotation des tomes publiés par Actes Sud entre 2006 et 2007. Ce titre secondaire (Millénium 1, 2 et 3) affiche clairement la logique sérielle dans laquelle s'inscrit la publication, avec trois volumes effectifs et beaucoup d'autres en projet. La segmentation du récit livré en épisodes successifs, et d'autre part, l'objet même de la fiction - un magazine par définition périodique - crée un effet de mimétisme sur le plan de l'énoncé et de l'énonciation : l'histoire racontée, mais également la manière de la raconter (Benveniste, 1966), situent le roman dans la périodicité et le continuum propre à la temporalité médiatique, sous la forme d'une saga dont le titre récurrent met en exergue la dimension collective du journalisme.

Selon la sémiotique narrative que nous mobilisons ici, la construction du personnage s'effectue dans le récit sur deux niveaux : le niveau sémantique et actoriel qui concerne les qualifications et les rôles thématiques attribués au personnage-acteur, et le niveau actantiel qui concerne la logique d'action analysée à partir des pôles actantiels et des programmes narratifs ${ }^{10}$. Ce sont ces deux plans que nous utilisons pour analyser, d'une part, la caractérisation de la revue saisie en tant que personnage-acteur et, d'autre part, la programmation

demokraterna från insidan [La Démocratie suédoise vue de l'intérieur], avec R. Slätt, M. Blomquist et D. Lagerlöf, Hjalmarson \& Högberg, Stockholm, 2004. Il collabore avec C. Englund pour Debatten om hedersmord. Feminism eller rasism [Débat sur le meurtre d’honneur. Féminisme ou racisme], Stockholm, Svartvitts, 2004 et «Violences suédoises et non suédoises contre les femmes », Stockholm, Svartvitts et Expo, 2004.

9. On pense notamment à son échec au concours d'entrée à l'école de journalisme, à sa carrière à Tidningarnas Telegrambyra et aux difficultés d'Expo, qui sont mentionnés dans la biographie de la compagne de l'auteur. «Finalement, comprenant qu'il ne progresserait jamais à Tidningarnas Telegrambyra, Stieg a choisi d'accepter les indemnités de départ et de se faire licencier en 1999. » (Gabrielson, 2011, p. 47)

10. Voir Greimas et Courtés, 1979, ou Adam, 1994 (chapitre 4: "La sémiotique narrative»). Le modèle actanciel comporte six actants : Destinateur/Destinataire, Sujet/Objet, Adjuvant/Opposant. Nous retiendrons ici principalement le couple Sujet/Objet et le couple Adjuvant/Opposant œuvrant dans la quête de vérité. 
narrative dans laquelle s'insère la revue saisie en tant qu'actant. Le magazine n'occupe pas systématiquement le devant de la scène dans la mesure où de nombreuses digressions dans l'enchâssement narratif l'éclipsent ponctuellement, mais toutes les intrigues renvoient in fine au sort de Millénium.

Mensuelle, la « revue d'investigation sociale et économique» se fonde sur un journalisme d'enquête scrutant en premier lieu des faits ordinaires, voire des faits divers, qui s'inscrivent finalement dans des dysfonctionnements politiques, économiques, judiciaires, sociétaux. Cette conception de l'enquête, qui rappelle celle des muckrackers étasuniens de la fin du Xıxe siècle, s'articule à un impératif d'exhaustivité qui implique une restitution dans un format long. En tant que revue dédiée, Millénium rassemble les caractéristiques idéales et se présente sous « une forme graphique moderne et attirante ». Le rôle thématique de «diseur de vérité insolent et fiable» est incarné par une équipe restreinte composée d'une directrice de publication et d'un rédacteur en chef, ainsi que d'un directeur artistique à temps partiel. «À part eux trois, Millénium consistait en deux collaborateurs à plein temps, trois personnes à temps partiel et un intérimaire» (t. I, p. 73). Installée dans des locaux de taille modeste mais bien situés au centre de Stockholm, la rédaction s'apparente à un microcosme plutôt intime, entre lieu de travail et lieu de vie. Le credo small is beautifull qui caractérise Millénium se traduit également par son modèle économique, garant de l'indépendance éditoriale. Tirant à 21000 exemplaires mensuels, l'entreprise de presse fonctionne sur un double marché lecteurs/annonceurs fidélisé, en visant l'équilibre financier plus que la rentabilité à outrance, et sur un actionnariat maîtrisé par l'équipe de direction de la rédaction. Erika Berger et Mikael Blomkvist, cofondateurs et copropriétaires de la publication, se révèlent journalistes-entrepreneurs en prise avec la gestion économique et organisationnelle du journal. Minutieusement décrite dans le roman, cette dimension entrepreneuriale et économique n'est pas dissociée de l'idéal journalistique porté par la revue d'investigation: «C'était le genre de périodique dont les comptes sont toujours dans le rouge, mais très prestigieux et où les collaborateurs adoraient travailler» (t. I, p. 73).

Le roman fictionnalise le journalisme du xxıe siècle sous les traits d'une petite structure, mais dotée d'un capital symbolique important, portée par deux journalistes passionnés mais également gestionnaires. La construction du personnage Millénium s'effectue par touches successives avec des qualifications portant sur sa notoriété (du type «un magazine connu pour son indépendance impertinente », t. I, p. 271), ou des passages décrivant très concrètement le fonctionnement collectif d'une revue et les différentes facettes de la production d'informations, de la conférence de rédaction jusqu'à la mise en page et l'impression. Cette construction s'effectue également en positionnant la revue dans le champ médiatique suédois, notamment par rapport aux médias mainstream réels ou imaginés. Plutôt que d'établir un parallèle avec 
des publications rentrant dans la même catégorie, la narration organise une comparaison entre le mensuel Millénium et Svenka Morgon-Posten, un quotidien fictif représentant la presse nationale de référence. Ce couple oppositionnel développé dans le troisième tome permet de mettre en miroir le journalisme pratiqué dans un petit magazine spécialisé et le journalisme pratiqué dans un grand quotidien d'information générale. L'opposition des valeurs se greffe sur le point de vue du personnage d'Erika Berger qui expérimente le poste de rédactrice en chef du quotidien après avoir dirigé la revue. Les péripéties dans lesquelles elle affronte de nombreuses difficultés renvoient aux relations de travail, à l'exercice hiérarchique, au traitement de l'information, à la déontologie ou encore à la financiarisation du journal, autant d'éléments multipliant les effets de réel et organisant une axiologie opposant le fonctionnement de la revue (pôle positif) à celui du grand quotidien (pôle négatif).

Sur le plan de la logique des actions, Millénium est soumis à des épreuves qui contribuent à définir le personnage comme entité collective. Les deux événements narratifs que constitue le départ des deux cofondateurs (Mikael Blomkvist dans le premier tome, Erika Berger dans le troisième) confrontent le magazine à des crises successives. Dans le deuxième tome, la privation, ou disjonction narrative, provoquant la déstabilisation de l'équipe concerne la mort d'un collaborateur. Dans tous les cas, "sortir» le magazine en temps et en heure devient un défi à surmonter, qui a pour corollaire le maintien d'un équilibre éditorial, organisationnel ou financier. Millénium mène une lutte permanente pour exister. Dans le premier volume, il s'agit de survivre au départ de Mikael Blomkvist à la suite d'une accusation en diffamation. Les répercussions de cette condamnation, qui entache l'honneur journalistique du héros (Ringoot, 2010), menacent la crédibilité du journal et provoquent la fuite des annonceurs. Dans le deuxième tome, il s'agit de surmonter le traumatisme lié au meurtre d'un collègue et de reprendre en main son enquête dont le contenu fait l'objet d'un numéro en cours. Dans le dernier opus, il s'agit de conjurer le désordre engendré par le départ de la directrice, garante du bon fonctionnement de l'équipe. Plus globalement, dans le programme narratif de base qui est celui de la quête de vérité, Millénium affronte de nombreux opposants qui ont intérêt à ce que les enquêtes ne soient pas publiées. Cette lutte permanente implique tous les membres de la rédaction et si les deux fondateurs de la revue se permettent de quitter tour à tour leur créature, ils reviennent néanmoins en son giron. Les défis relevés collectivement participent à forger l'unité du personnage, qui est également exposé à une transformation susceptible d'affecter son identité. Amorcé à la fin du premier volume, le projet d'étendre l'activité de la revue à celle de maison d'édition est développé dans les tomes suivants. La diversification de la production dont il est question s'inscrit dans la logique éditoriale du mensuel en ouvrant la publication d'enquête au support livre. 


\section{Le journaliste et le livre}

La dichotomie entre l'auteur collectif incarné par Millénium et l'auteur individualisé incarné par Mikael Blomkvist s'opère notamment à partir d'un ressort narratif remarquable dans les trois tomes, celui du « livre dans le livre». Car finalement, une enquête journalistique publiée ou publiable dans les pages du magazine devient toujours un livre signé par le journaliste. L'intrigue portant sur des meurtres en série développée dans le premier tome fait apparaître, en toile de fond historique, les accointances nazies d'une grande famille de l'industrie suédoise remontant à la période d'avant-guerre. Les ramifications narratives permettent également de traiter le thème de la délinquance en col blanc, incarnée par un chef d'entreprise «véreux» détournant de l'argent public dans des montages financiers. Ce premier roman s'achève sur la publication d'un livre consacré à cette "affaire Wennerström» et intitulé Le banquier de la mafia, dans lequel le héros journaliste dénonce les dérives du libéralisme économique et du capitalisme financier. Dans les tomes suivants, le thème du livre journalistique occupe une place encore plus importante dans la mesure où la narration s'organise autour d'un livre dont la publication correspond à l'aboutissement du récit.

À l'instar du Manuscrit trouvé à Saragosse ${ }^{11}$, de nombreux romans ont mis au cœur de leur intrigue un livre, mystérieux, perdu ou à venir, à partir duquel des aventures s'enchevêtrent. Le deuxième tome de Millénium s'inscrit dans cette catégorie, à la différence que, dans ce cas, il s'agit de compléter et de publier un livre d'enquête inachevé, écrit par un journaliste victime de meurtre. L'histoire de ce livre dans le livre débute dans la deuxième partie («Bons baisers de Russie», p.79), par la négociation d'une pige proposée au magazine, qui se greffe sur un projet plus ambitieux :

Le trafic de femmes, dit Dag Svensson. C'est-à-dire l'exploitation sexuelle des femmes. Dans le cas présent, principalement originaires des pays baltes et de l'Europe de l'Est. Pour tout vous dire, je suis en train d'écrire un livre là-dessus, c'est pour cela que j'ai contacté Erika - vu que vous fonctionnez aussi comme maison d'édition... (t. II, p. 95-99)

La collaboration se conclut durant un échange réaliste alternant questions de fond (documentation, sources, méthodes), ajustements pratiques (contractualisation, rémunération) et prévisions organisationnelles (secrétariat de

11. Écrit au xvııı siècle par Jean Potocki et considéré comme le chef-d’œuvre du roman à tiroir, le Manuscrit trouvé à Saragosse est publié pour la première fois en France en 1958 par Roger Caillois chez Gallimard. Pour les différentes versions et traductions, voir la présentation du travail d'édition de Dominique Triaire et François Rosset sur http://www2.cnrs.fr/journal/3387.htm (consulté le 8 décembre 2013), «La saga du Manuscrit trouvé à Saragosse ». 
rédaction, édition, mise en page). Incriminant des «michetons» flics, avocats, procureurs ou journalistes dans des actes de violence sur des prostituées éventuellement mineures, ainsi qu'une mafia du sexe composée de «crétins brutaux peu organisés», l'enquête veut dénoncer l'indifférence politique, judiciaire et policière à l'égard de victimes au plus «bas de l'échelle sociale». Le meurtre du journaliste et de sa compagne criminologue intervient au tout début de la troisième partie du roman (t. II, p. 213), provoquant alors une démultiplication d'enquêtes racontées : celle de la police, celle des médias suédois qui donne lieu à une critique portant sur le suivisme et le sensationnalisme médiatique, celle des journalistes de Millénium qui explorent des pistes différentes. Pour les collaborateurs de la revue, le livre inachevé devient alors l'enjeu d'une double quête : l'aboutissement du travail journalistique interrompu et la recherche du meurtrier du collègue. À la fin du tome II, tous les éléments sont réunis pour accréditer la thèse que l'enquête consacrée à la prostitution est à l'origine de l'élimination du journaliste dont les révélations menacent les protagonistes incriminés. La dénonciation d'une affaire de mœurs se transforme en dénonciation d'une affaire d'État, avec pour noyau commun les agissements des membres d'un groupe occulte formé au sein des services de sécurité (Säpo).

Le rebondissement de l'enquête qui touche au secret d'État développé dans le dernier tome amène Mikael Blomkvist à concevoir le projet d'un autre livre :

Il s'était obstiné à continuer à travailler sur les textes avec lesquels il avait l'intention de constituer le numéro d'été de Millénium, mais avait fini par se rendre compte que son matériel était si vaste que même un numéro thématique n'y suffirait pas. Confronté à la même situation que pour l'affaire Wennerström, il avait décidé de publier ses textes sous forme de livre. (t. III, p. 231)

Les enchaînements narratifs qui structurent le récit de Millénium 3 aboutissent à la sortie conjointe des deux livres, en même temps que se dénoue le suspense autour du procès de Lisbeth Salander, présumée coupable de plusieurs meurtres. Dans l'économie du récit, le procès représente l'événement vers lequel tendent deux programmes narratifs opposés : l'un consistant à faire la preuve de l'innocence de l'accusée, l'autre consistant à faire la preuve de sa culpabilité. Dans la quête de vérité qui aboutit à la réhabilitation de Lisbeth Salander, le héros Mikael Blomkvist n'est pas seul. Les deux anti-programmes organisent l'antagonisme des différents personnages, ceux qui œuvrent à prouver le statut de victime de la jeune femme contre ceux qui la veulent coupable, avec, dans les deux camps, des représentants des institutions impliquées. Cependant, c'est le livre de Mikael Blomkvist qui contribue en premier lieu à réhabiliter Lisbeth Salander, en révélant l'existence et les exactions de « la section » secrète de la Sapö.

L'histoire de Lisbeth Salander, consubstantielle à celle de Millénium et de Mikael Blomkvist, cristallise le dysfonctionnement des institutions judiciaire, 
policière, sociale, éducative et médiatique, dont la révélation constitue l'idéal journalistique construit au fil des quelque 1900 pages de la trilogie. Dans celleci, l'imbrication de l'engagement idéologique et de l'engagement professionnel s'effectue par la dénonciation du sexisme, de l'extrême droite, du secret d'État et plus généralement des injustices et des discriminations. Le héros de Millénium corrobore la conclusion que Denis Ruellan propose à l'issue de sa catégorisation des figures journalistiques dans la fiction :

L'engagement est le système, il n'est pas le problème. Le problème, c'est de croire que l'on puisse être journaliste sans s'engager : dans son métier, dans l'histoire, dans la vie des autres, dans des relations plurielles, dans sa vie, la coexistence de toutes ces facettes exigeant des ajustements fins et toujours particuliers. (Ruellan, 2010, p. 29)

L'engagement de Mikael Blomkvist se traduit en termes d'illusio ${ }^{12}$ professionnelle qui a pour corollaire la pratique de l'enquête. C'est également la définition de l'information politique qui est ici en jeu, dans le sens où le rôle du journaliste consiste à éclairer et à mesurer les effets de l'exercice politique et des fonctionnements institutionnels sur la vie ordinaire du citoyen/administré. Peu compatible avec la division du travail journalistique et la structuration par rubriques que connaît la presse généraliste et politique dominante, cette vision transversale du politique correspond à des projets éditoriaux alternatifs. La revue Millénium défend la vision d'un journalisme engagé sans pour autant se présenter comme une publication militante.

L'enquête journalistique pensée en fonction de deux supports (périodique et livre) s'inscrit dans le modèle du journalisme d'investigation américain tel que présenté par Marc Lee Hunter dans son manuel préconisant aux journalistes d'envisager la publication de la story sous forme d'article ou sous forme de livre (Hunter, 2011). Cependant, la fictionnalisation conjointe de la revue et du livre prône davantage une formule bi-média qui redistribue les dimensions collective et individuelle de l'auctorialité journalistique. Le numéro de la revue et le livre qui y est associé dépendent tous deux d'une organisation collective du travail. Dans le cas de l'enquête du journaliste assassiné, plusieurs collaborateurs sont affectés à la réalisation du livre dès le démarrage du projet (t. II, p.100-102) et bien évidemment dans sa finalisation posthume. Dans le cas de la publication de "La section» (t. III), Mikael Blomkvist s'adjoint l'assistance de collaborateurs dans la phase de recherche ainsi que dans la phase de rédaction et d'édition. Le double projet éditorial a également pour effet de niveler la temporalité de l'écriture et de la publication, en conjuguant les modalités de la

12. Notion «qui renvoie à un investissement psychique, intellectuel et professionnel dans les jeux et les enjeux propres à un champ [...] Une illusio qui se cristallise dans la vision du journaliste comme celui qui, selon le mot d'Albert Londres, "porte la plume dans la plaie" fera merveille dans le journalisme d'investigation. » Neveu, 2013 (4e réédition). 
production d'un magazine et celle de la production d'un livre. Le héros note à cet égard: «Je n'ai jamais compris pourquoi les éditeurs ordinaires exigent dixhuit mois de délai de production pour sortir quelques centaines de pages. Il faut une semaine pour faire la mise en page [...] et deux semaines pour imprimer » (t. III, p.100). La publication bi-média provoque des tensions dans l'équipe, exacerbées par la surcharge de travail, mais également par le comportement du héros parfois jugé trop individualiste. Et finalement, s'ils portent fièrement la griffe de Millénium, les livres n'en sont pas moins médiatisés et valorisés en nom propre par Mikael Blomkvist. L'héroïsation du journaliste auteur de livre relève d'un modus operandi récurrent puisque, dans le premier volume comme dans le dernier, Mikael Blomkvist fait l'objet d'une édition spéciale sur une chaîne de télévision, suivie d'une consécration médiatique nationale.

\section{La hackeuse et le journaliste}

Le personnage de Lisbeth Salander occupe plusieurs pôles actanciels dans le roman, notamment celui de sujet dans les programmes narratifs concernant ses propres enquêtes pour le compte de l'entreprise de sécurité qui l'emploie, et celui d'objet dans les programmes narratifs concernant les enquêtes sociales, policières ou journalistiques dont elle est l'enjeu. Eu égard à notre objectif qui consiste à étudier la représentation du journalisme, nous retiendrons ici plus particulièrement le rôle d'adjuvant qui lui est conféré dans le cadre des investigations menées par ou avec le héros Mikael Blomkvist. Collaborant à plusieurs reprises, le tandem renouvelle le couple oppositionnel journaliste/détective récurent dans la fictionnalisation du journalisme. Les objectifs et les méthodes d'enquête du journaliste ainsi que les questions déontologiques afférentes développées dans le roman s'organisent non plus en fonction des pratiques connexes de l'inspecteur de police, du juge d'instruction ou du simple détective privé, mais en fonction de celles du hackeur (hacker). En ce sens, la fiction de Millénium devance la consécration du hackeur dans la réalité, telle celle de Julien Assange déclaré «homme de l'année » par le journal Le Monde en 2010. En revanche, le roman n'opère pas la fusion de la figure du hackeur et de la figure du journaliste dans la mesure où deux héros distincts prennent en charge l'une et l'autre. Cette dichotomie entre journalisme et hacking différencie les deux activités, mais elle les situe également dans un rapport de réciprocité. Les investigations collaboratives menées par les deux personnages mêlent l'enquête de terrain et la recherche d'indices dans le cyberespace.

Bien que collectées de manière illégale, les informations que Lisbeth Salander transmet au journaliste permettent d'apporter les preuves nécessaires à la révélation de crimes impliquant plus ou moins directement des membres de l'élite sociale : criminalité financière, pédophilie, complot politique, trafics, 
prostitution, etc. Toutes les affaires révélées par Mikael Blomkvist s'articulent sur le piratage de données performé par la hackeuse. Capable de s'introduire dans les systèmes les plus sophistiqués, Lisbeth Salander cumule le statut de collaboratrice et celui de «source». L'espionnage de comptes bancaires, de courriers électroniques, de sites consultés ou encore de documents stockés sur les ordinateurs personnels ou professionnels dévoile des renseignements inaccessibles au commun des journalistes. Refusant de préciser à ses collègues par quel canal il obtient ces informations, le journaliste admet mener "une activité criminelle d'envergure»: «Ce sont pour ainsi dire les formes de ma recherche qui sont un peu douteuses » (t. I, p. 525). Pour utiliser les éléments piratés, Mikael Blomkvist actionne le levier de la "protection des sources» qui lui octroie le droit de rester discret sur la provenance des informations et l'identité de l'informateur. À la fin du premier tome, le paradoxe qui consiste à légitimer la pratique illégale du hacking par le biais du secret des sources pose quelque problème éthique au héros : «On le décrivait comme un héros en termes si flagorneurs qu'il en fut gêné» (t. l, p. 558). Encensé par ses pairs qui le comparent aux journalistes américains «qui préfèrent aller en prison plutôt que de révéler une source », Mikael Blomkvist est assimilé aux illustres journalistes du Washington Post à l'origine de l'affaire du Watergate. La transposition vaut également pour la source présentée comme la "Gorge profonde» du héros. La référence au Watergate, convoquée à plusieurs reprises dans le récit, alimente l'héroïsation des enquêteurs en activant un modèle journalistique qui a acquis le statut de mythe professionnel.

Le couple oppositionnel journaliste/hackeuse fictionnalise une nouvelle dimension du journalisme qui interroge la production et la divulgation de l'information à l'ère d'Internet. Dans le rôle d'adjuvant, la hackeuse permet au journaliste de réaliser sa mission d'intérêt général qui consiste à rendre public les dysfonctionnements de la démocratie. Le hacking œuvre pour la bonne cause et pour la cause journalistique qui se superposent dans le roman. Millénium contribue à valoriser ce que Sylvain Parasie nomme le « journalisme "hacker" » en le rapportant à «une nouvelle utopie pour la presse» (Parasie, 2011). Le terme hacker renvoie ici aux compétences technologiques utilisées dans un cadre légal par les programmeurs/journalistes de la presse américaine étudiés par le sociologue :

Régulièrement, des journalistes prennent à témoin l'opinion publique en pointant le comportement immoral des élites et des gouvernants. Que ce soit sous la figure du publiciste français du Xvııe siècle, du muckracker américain du début du $x x^{e}$ siècle ou de la figure plus récente du journaliste d'investigation, ces journalistes veulent rendre visible ce qui est ordinairement soustrait à la vue des citoyens, de manière à contraindre les gouvernants. Tout en venant d'horizons sociaux très différents, certains programmeurs/journalistes ont à la fois prolongé et donné un tour particulier à cette posture. (Parasie, 2011) 
Si ces valeurs journalistiques caractérisent également le héros du roman, ce dernier ne possède pas pour autant les qualifications informatiques.

La dualisation journaliste/hackeur maintient une barrière entre les deux et fictionnalise le hacking dans d'autres univers que celui du journalisme. Utilisant ses compétences dans des investigations menées à titre professionnel pour une entreprise de sécurité, ou menées à titre personnel pour son propre combat idéologique, Lisbeth Salander est membre d'un cercle très fermé : «La République des hackers». Le personnage s’inscrit dans la lignée des héros rebelles de la contre-culture, qui règlent leurs comptes avec la société par leurs propres moyens. Cette dimension s'écarte de notre propos, mais donne souvent matière à développer des thèmes journalistiques au sein du roman. La question des paradis fiscaux, notamment, est traitée par le biais d'un détournement d'argent perpétré par la hackeuse au détriment du «financier véreux» évoqué plus haut. La séquence (t. I, p. 547-554) portant sur le voyage de la jeune femme à Zurich explique par le menu différentes opérations visant à occulter le circuit de l'argent : transfert de comptes domiciliés aux îles Caïman via les services d'une banque suisse, ouverture d'un compte dans une autre banque au titre d'une "société bidon particulièrement anonyme à Gibraltar », lequel compte est ensuite vidé « pour régler des factures d'un grand nombre de sociétés bidons partout dans le monde» (t. l, p. 551), et rapatriement final des fonds sur un nouveau compte créé aux îles Caïman. L'insertion d’éléments informatifs concernant des problèmes publics réels médiatisés par les journalistes constitue un procédé récurent dans le roman. Les passages consacrés à l'explication d'un fait de société non fictionnel sont intégrés dans la narration, mais acquièrent une forme d'autonomie par leur visée explicative et informative propre au discours journalistique. C'est également le cas pour les éléments d'information portant sur l'histoire politique de la Suède et le fonctionnement de ses institutions qui font l'objet de développements. Celui qui concerne la Sapö est particulièrement renseigné (t. III, p. 286-290) et comporte même une citation du texte de loi définissant sa mission de «Protection de la Constitution ». L'insertion du discours journalistique dans le discours littéraire offre une autre facette de la «culture médiatique» à laquelle Millénium contribue.

Récit œuvrant à la gloire du journalisme d’investigation, Millénium engendre une co-énonciation littéraire (Maingueneau, 2009) alimentée par les journalistes et le secteur de l'édition. Le décès de Stieg Larsson avant même la publication du roman a contribué à construire une image d'auteur atypique quand bien même d'autres cas semblables lui préexistent. Cette configuration dramatique dans laquelle l'écrivain n'a pas pu assister au succès de son œuvre a provoqué une prolifération d'énoncés qui tendent à héroïser l'auteur du roman. Encore au Nouvel Observateur à l'époque, Florence Aubenas constatait que «dans "Millénium", l'assassin c'est le livre» dans un article intitulé «Com- 
ment “Millénium" m'a envahie »13. D'autres facteurs ont entretenu la rumeur médiatique, dont la controverse portant sur l'authenticité du troisième tome, ou encore l'existence d'un quatrième tome inachevé. Guillaume Lebeau, auteur de romans policiers, publie en 2008 un livre consacré au «mystère du quatrième manuscrit» annoncé comme "une enquête au cœur de la série "Millénium" » et collabore un peu plus tard à la réalisation d'une bande dessinée portant sur Stieg Larsson ${ }^{14}$. L'affaire judiciaire opposant la famille de l'auteur à sa conjointe quant à l'héritage symbolique et matériel du travail de Stieg Larsson a également fait perdurer les discours journalistiques consacrés à Millénium et son auteur. Actes Sud, à nouveau, publie en 2011 la biographie d'Eva Gabrielson (2011), compagne du journaliste-écrivain, qui fustige la «mythification» du "Stieg de Millénium» ainsi que «l'industrie Millénium». Enfin, le film suédois diffusé en 2009 et adapté par UGC/TF1, la série de six épisodes diffusée en 2010 sur Canal + France ainsi que le film de David Fincher sorti en France en janvier 2012 ont maintenu Millénium dans l'actualité journalistique. Le roman et son auteur sont devenus des objets de discours circulant dans l'espace public bien au-delà des « retombées presse » attendues dans le cadre du marketing d'édition.

Centré sur le récit romanesque, notre propos visait à analyser comment Millénium s'insère dans la culture journalistique en questionnant les procédures de fictionnalisation du journalisme. Pour les spécialistes du lien entre littérature et journalisme, c'est le « reporter fictif qui a porté le mythe du reporter triomphant» (Boucharenc, 2011) avant que les journalistes réels se mettent en scène dans leurs livres racontant leurs reportages et enquêtes. Dans Millénium, le personnage de Mikael Blomkvist perpétue la tradition littéraire du reporter-enquêteur, mais son héroïsation convoque des références non fictionnelles, notamment celle de Bob Woodward. Si le mécanisme de préfiguration du réel dans la fiction fonctionne dans Millénium, c'est plutôt par le biais de la hackeuse fictive, qui préfigure la consécration à venir du journalismehackeur. Écrit avant l'émergence du site Wikileaks qui promeut la «fuite» et la publication de documents institutionnels confidentiels, et a fortiori avant les révélations de l'Offshore Leaks ${ }^{15}$ sur l'évasion fiscale, Millénium envisage néanmoins les avantages et les inconvénients du mariage entre hacking et journalisme. L'autre aspect remarquable est celui de l'extension du périmètre journalistique dans le secteur de l'édition. Dans le registre particulier de l'enquête ou de l'investigation, les livres constituent un support privilégié pour le

13. Publié le 14 février 2008, en ligne : http://bibliobs.nouvelobs.com/romans/20080214. BIB0792/comment-millenium-m-039-a-envahie.html (consulté le 8 novembre 2013).

14. G. Lebeau, Le mystère du quatrième manuscrit. Enquête au cœur de la série "Millénium », Paris, Du Toucan, 2008 et G. Lebeau et F. Rébéna Frédéric, 2012, Stieg Larsson avant Millénium, Paris, Denoël Graphic.

15. Enquête de l'International Consortium for Investigative Journalism sur la fraude fiscale et les paradis fiscaux révélée en 2013. 
journalisme de révélation, notamment dans le domaine politico-judiciaire. La fiction apporte toutefois un regard particulier sur la double auctorialité journalistique en brouillant les frontières qui séparent ce qui relève du journal et ce qui relève du journaliste. Dans Millénium, le livre s’insère dans un projet à court terme et dans un processus de diffusion qui intègrent la logique événementielle du périodique. Le livre garantit la spécificité du registre de discours tenu en nom propre, mais porte aussi la marque du collectif. C'est un fonctionnement assez similaire qu'adopte le pure player français Médiapart dans le traitement de «l'affaire Cahuzac ${ }^{16}$. Érigé en personnage à part entière, le magazine fictif Millénium incarne les valeurs d'un journalisme somme toute artisanal, émanant d'une petite structure resserrée autour de sa mission de " diseur de vérité ». Dans cette configuration, la dualisation des acteurs narratifs collectif/individuel accentue le tiraillement entre identité éditoriale et individuation auctoriale, par l'héroïsation des deux figures.

\section{Références}

ADAM Jean-Michel, 1994, Le récit, Paris, Presses universitaires de France.

BenVEnISte Émile, 1966, Problèmes de linguistique générale, Paris, Gallimard.

BOUCHARENC Myriam, 2011, « Nouvelles fictions du reporter au xxe siècle », Interférences littéraires / Literaire interferenties, nº 7, p. 115-125.

Eco Umberto, 1981, «James Bond, une combinatoire narrative», L'analyse structurale

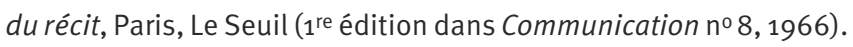

Gabrielson Eva (et Colombanı Marie-Françoise), 2011, Millénium, Stieg et moi, Arles, Actes Sud (Babel).

GreImAS Algirdas Julien, Courtés Joseph, 1979, Sémiotique. Dictionnaire raisonné de la théorie du langage, Paris, Hachette.

HUNTER Mark Lee, 2011, L'enquête par hypothèse. Manuel du journaliste d'investigation, en ligne: http://markleehunter.free.fr/ (consulté le 8 décembre 2013).

Maingueneau Dominique, 2009, "Auteur et image d'auteur en analyse du discours», Argumentation et analyse du discours, nº 3 , en ligne : http://aad.revues.org/660

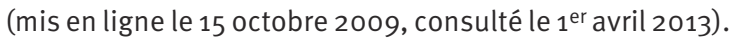

NeVEu Erik, 2013, Sociologie du journalisme, Paris, La Découverte (4 ${ }^{\mathrm{e}}$ réédition).

PARASIE Sylvain, 2011, « Le journalisme "hacker”. Une nouvelle utopie pour la presse? », La vie des idées, en ligne : http://www.laviedesidees.fr/Le-journalisme-hacker. html (consulté le 8 décembre 2013).

PINSON Guillaume, 2010, "Le reporter fictif (1863-1913)», Autour de Vallès. Revue de lectures et d'études vallésiennes, $\mathrm{n}^{\circ}$ 40, p. 87-99.

16. Médiapart révèle en décembre 2012 que Jérôme Cahuzac détient un compte non déclaré en Suisse. Fabrice Arfi publie en mars 2013 L'affaire Cahuzac, en bloc et en détails (Paris, Don Quichotte). Sur la première de couverture, le nom de l'auteur est suivi de la mention « avec la rédaction de Médiapart». 
- 2012, «L'imaginaire médiatique. Réflexions sur les représentations du journalisme au xIxe siècle », COnTEXTES, no 11, en ligne : http://contextes.revues.org/5306 (mis en ligne le 16 mai 2012, consulté le 31 mars 2013).

Ringoot Roselyne, 2010, «Formes romanesques de l'éthique journalistique. Millénium, un magazine et des journalistes entre réalité et fiction», Les journalismes. Réalités plurielles, éthiques communes? Chaire de recherche en éthique du journalisme, Ottawa, http://crej.ca/publications/journalismes.html (consulté le 8 décembre 2013).

- 2012, "Le journalisme entre auctorialité et discours», Habilitation à diriger des recherches soutenue le 3 décembre 2012, Université Rennes 1.

RuelLAN Denis, 2010, "Des reporters en plein paradoxe. Représentation au cinéma (1975-1988) », S. Lévêque, D. Ruellan éd., Journalistes engagés, Rennes, Presses universitaires de Rennes, p. 17-27.

\section{Corpus}

LARSSON Stieg, 2006a, Les hommes qui n'aimaient pas les femmes, Millénium 1, Arles, Actes Sud.

- 2006b, La fille qui rêvait d'un bidon d'essence et d'une allumette, Millénium 2, Arles, Actes Sud.

— 2007, La reine dans le palais de courants d'air, Millénium 3, Arles, Actes Sud. 